\title{
A simple, analytical model of collisionless magnetic reconnection in a pair plasma
}

\author{
Michael Hesse, Seiji Zenitani, Masha Kuznetsova, and Alex Klimas \\ All at: Space Weather Laboratory, Code 674, NASA Goddard Space Flight Center, \\ Greenbelt, Maryland, 20771, USA
}

\begin{abstract}
A set of conservation equations is utilized to derive balance equations in the reconnection diffusion region of a symmetric pair plasma. The reconnection electric field is assumed to have the function to maintain the current density in the diffusion region, and to impart thermal energy to the plasma by means of quasi-viscous dissipation. Using these assumptions it is possible to derive a simple set of equations for diffusion region parameters in dependence on inflow conditions and on plasma compressibility. These equations are solved by means of a simple, iterative, procedure. The solutions show expected features such as dominance of enthalpy flux in the reconnection outflow, as well as combination of adiabatic and quasi-viscous heating. Furthermore, the model predicts a maximum reconnection electric field of $E^{*}=0.4$, normalized to the parameters at the inflow edge of the diffusion region.
\end{abstract}




\section{INTRODUCTION}

Magnetic reconnection is one of the most fundamental plasma processes in laboratory, space, and astrophysical plasmas. Reconnection operates by converting magnetic energy into particle energy, which manifests itself in form of heat or pressure, and in form of bulk motion ${ }^{1}$. Reconnection also facilitates plasma transport across topological magnetic boundaries, and it may change the overall topology of the magnetic field ${ }^{2}$.

Reconnection is a meaningful concept only if plasma component species are frozen into the magnetic field in the vast majority of the physical volume under consideration. If this condition is not fulfilled the system is referred to as diffusive. In the former case, reconnection is facilitated by a localized region, wherein the frozen-in condition is violated. This region is usually termed the diffusion region.

A further distinction concerns the processes effecting the violation of the frozen-in condition in the diffusion region. In a collisional plasma, such as found in some laboratory experiments, the lower ionosphere of the Earth, the lower solar atmosphere and in some astrophysical plasmas, inter-particle collisions effectively scatter particles off magnetic field lines. Macroscopically, collisional processes of this kind heat the plasma, and they produce a resistivity.

In a collisionless plasma, the means of demagnetizing particles is not as immediately obvious. Since most space plasmas and many laboratory plasmas fall into this category, this question has received considerable attention as a research focus, primarily based on 
numerical simulations, and also by data analyses of laboratory and space measurements. Candidate processes include wave-particle interactions, as well as particle inertia-based processes. Numerical simulations exhibit evidence for both, and a resolution of which process dominates when remains outstanding.

Owing to the complexity of the problem, the vast majority of kinetic reconnection research has been based on modeling, with accompanying theoretical estimates. Analytical theory has focused primarily on MHD, Hall-MHD, or multi-fluid studies ${ }^{3-8}$, some of which assume, for simplicity, a resistive evolution in the diffusion region. A recent study employed a viscous term ${ }^{7}$ in a pair plasma model to generate analytical estimates for diffusion region properties. While these studies have been extraordinarily successful, they do not fully address the kinetic nature of the diffusion region.

Numerical simulations of pair plasmas have provided ample evidence of fast reconnection, and dissipation has been most commonly attributed to pressure tensor effects $^{9-12}$. There is, however, disagreement in the literature as to why reconnection remains fast even in the absence of Hall-type effects.

A significant step toward an analytical, kinetic theory of collisionless magnetic reconnection has recently been undertaken by Tsiklauri ${ }^{13}$. This work recognizes the relation between the reconnection electric field and the nongyrotropic components of the electron pressure tensor. By assuming incompressibility, the authors show that fast reconnection may result from reasonable assumptions of the extent of the diffusion region.

In this paper, we provide a complementary step toward a kinetic model of the diffusion region. We will adopt previous assumptions and theory results ${ }^{13}$, such as a 
diffusion region width given by particle bounce motions and pressure tensor-based dissipation, which appears to be consistent with numerical models. We will, however, primarily use conservation-based arguments for both momentum and energy to derive equations, which specify the dimensions of the diffusion region completely, and which therefore yield estimates of the reconnection rate.

In order to proceed with this theory, we adopt a number of assumptions, some of which (1-3) are basic requirements for magnetic reconnection. Some further assumptions (4-11) are adopted to simplify the problem. These are:

Basic properties of magnetic reconnection: the role of the reconnection electric field

1. The first function of the reconnection electric field is to sustain the current density in the diffusion region. Incoming plasma typically does not exhibit drift velocities, which support the current flow required by the external magnetic field. At the same time, losses through convection and outflow reduce the current density. The reconnection electric field thus serves to accelerate the incoming plasma such that sufficient current is flowing in the diffusion region.

2. The second function of the reconnection electric field is to heat the incoming plasma populations such that pressure balance with the outside system is maintained. The reconnection electric field must have this role in order to replace the convective loss of hot plasma through the reconnection outflow.

3. Items 1) and 2) imply that the energy imparted by the reconnection electric field will manifest itself in part as bulk flow in the current direction for positively charged 
particles, and against it for electrons, and in part as increase of the plasma thermal energy.

We contend that items 1)-3) need to be fulfilled in any kinetic reconnection process. In addition to these axioms, we need to make a set of simplifying assumptions. These are:

4. We consider a symmetric, two-species, plasma, consisting of electrons and positrons only, with equal temperatures and densities. Extensions to more complex plasmas will be discussed but will remain a topic of future studies.

5. The plasma will be treated as polytropic, with small, nongyrotropic, deviations in the diffusion region. These deviations will be derived from a perturbation-type analysis.

6. The thickness of the diffusion region in-between the magnetic fields in the inflow region, is given by the particle bounce width.

7. The system is in steady state.

8. The system is translationally invariant in the out-of-plane direction.

9. Both sides of the inflow region are symmetric, and there is no guide field in the model.

10. Pressures, densities, and current densities in the diffusion region are very similar to the adjacent outflow region.

11. Heat flux effects in the energy equation are neglected. 
We will use these assumptions in an analysis of conservation equations, in particular of momentum and energy equations. In the next section, we will discuss preliminaries. Section III will focus on the momentum equation, and section IV will analyze the energy equation. Section $\mathrm{V}$ will combine all results to derive a complete set of equations for diffusion region properties. Section VI will discuss results, and section VII will provide a summary and discussion.

\section{PRELIMINARY CONCEPTS}

We assume that the diffusion region is a rectangular box of width $2 L$ and thickness $2 d$. Outside of this box, the plasma is assumed to be frozen into the magnetic field, whereas inside the plasma is demagnetized. Quantities at the inflow boundary are denoted by the subscript ' $\mathrm{i}$ ' and outflow boundary quantities are denoted by the subscript ' $\mathrm{o}$ '. Using assumption 10), we also denote by the latter subscript pressures and densities in the diffusion region.

We normalize all quantities in the following way: The magnetic field is normalized to its value at the inflow boundary of the diffusion region, $B_{i}$, the pressure by $B_{i}{ }^{2} / \mu_{0}$, the velocity by the single-species Alfven speed at the inflow boundary $v_{A i}=\frac{B_{i}}{\sqrt{\mu_{o} m n_{i}}}$, the electric field by the product $v_{A i} B_{i}$, the mass by the electron (or positron) mass, the density 
by its value at the inflow boundary $n_{i}$, and the length scale by an inflow boundary inertial length $L=\frac{c}{\omega_{i}}=c\left(\frac{e^{2} n_{i}}{\varepsilon_{o} m}\right)^{-1 / 2}$.

A standard continuity equation then relates, for each species, inflow and outflow densities, velocities, and dimensions:

$$
v_{i}=v_{o} n_{o} \frac{d}{L}
$$

The steady-state assumption implies that the reconnection electric field $\mathrm{E}^{*}$ equals the external electric field, both on the inflow and outflow side. This implies:

$$
E^{*}=v_{o} B_{o}=v_{i}
$$

Combining (1) and (2) leads to:

$$
B_{o}=n_{o} \frac{d}{L}
$$

\section{MOMENTUM EQUATION}

The momentum equation plays a critical role in this theory. Specifically, it will be applied to provide two estimates for current sheet width (subsections III A and III C), as well as one for the outflow velocity (subsection III B). These results will later be combined with an energy-equation-based derivation to obtain estimates of the reconnection rate. 


\section{A Current sheet pressure and sheet thickness}

Using assumption 6), the sheet thickness is given by the bounce width of thermal particles in the field reversal established by $B_{i}$. The bounce width, on the other hand, involves the thermal velocity of the particles in the diffusion region. The force balance in the $z$-direction determines the diffusion region thermal pressure, which equals the sum of inflow magnetic force, and plasma thermal and kinetic pressures:

$$
j_{y} B_{x}+2 \frac{\partial p}{\partial z}+2 \frac{\partial}{\partial z}\left(n v_{z}^{2}\right)=0
$$

where the factors of two reflect the combined pressures and velocities of both species. This is readily written as:

$$
\frac{1}{2} \frac{\partial B_{x}^{2}}{\partial z}-B_{x} \frac{\partial B_{z}}{\partial x}+2 \frac{\partial p}{\partial z}+2 \frac{\partial}{\partial z}\left(n v_{z}^{2}\right)=0
$$

Replacing derivatives by finite differences and integration over $z$ yields:

$$
\frac{1}{4}-\frac{1}{4} B_{o} \frac{d}{L}+p_{i}+v_{i}^{2}=p_{o}
$$

where $p_{o}$ is the constant value of the current sheet pressure. Using assumption 10) we find for the thermal velocity in the diffusion region:

$$
v_{t h, o}=\sqrt{\frac{2 p_{o}}{n_{o}}}
$$

In dimensionless units, the bounce width $d=\frac{m v_{t h, o}}{e B_{i}}$ becomes immediately: 


$$
d=\sqrt{\frac{2 p_{o}}{n_{o}}}
$$

This expression will be used below. In the next step, we analyze the force balance in both $x$ directions.

\section{B Outflow velocity}

The assumed symmetry of the particle populations implies that there is no electric field in the $x$-z plane ( $x$ denotes the outflow and $z$ the inflow direction). Using assumption 10), we neglect pressure gradients in the outflow direction. Accordingly, the only force capable of accelerating the plasma in the outflow direction is the Lorentz force. We therefore write the $x$-component of the momentum equation as follows:

$$
\left[2 \nabla \cdot\left(n_{o} v \mathcal{r} v\right)-\dot{j} \times \dot{B}\right] \cdot e^{r}=0
$$

where $j$ denotes the total current density. Note that the inertial contribution of each species balances one half of the total Lorentz force. Replacing derivatives by finite differences and using (3), the current density can be expressed as:

$$
j_{y}=2 n_{o} v_{y}=\left(\frac{1}{d}-\frac{B_{o}}{L}\right)=\left(\frac{1}{d}-n_{o} \frac{d}{L^{2}}\right)
$$


Insertion into (7) and integrating over $1 / 4$ volume of the diffusion region leads to:

$$
n_{o} v_{o}^{2} d-\frac{1}{2}\left(1-n_{o} \frac{d^{2}}{L^{2}}\right) \int d x B_{z}=0
$$

Assuming a linear variation of $B_{z}$ with $x$ and using (3) then yields:

$$
v_{o}=\frac{1}{2}\left(1-n_{o} \frac{d^{2}}{L^{2}}\right)^{1 / 2}
$$

We see that a smaller current density, resulting from an aspect ratio $d / L$ closer to unity, will lead to less acceleration and hence less outflow velocity. The inertia of larger current sheet density has a similar effect.

\section{C Force balance in the current direction}

Using assumption 5), we write the total pressure tensor as:

$$
\grave{P}=p \grave{1}+\grave{\Pi}
$$

where the second term denotes a small, nongyrotropic, contribution. Without loss of generality we perform this analysis for the electron species; the ion equations result from a simple change of signs. Thus, the $y$ component of the momentum equation becomes:

$$
-\nabla \cdot\left(n v_{y} v\right)=n\left(E_{y}+v_{z} B_{x}-v_{x} B_{z}\right)+\frac{\partial}{\partial x} \Pi_{x y}+\frac{\partial}{\partial z} \Pi_{y z}
$$


In the diffusion region, the electron pressure tensor components are well approximated by ${ }^{14}$ :

$$
\begin{aligned}
& \Pi_{x y}=-\frac{p_{o}}{B_{o}} \frac{\partial v_{x}}{\partial x} \approx-\frac{p_{o}}{B_{o}} \frac{v_{o}}{L} \\
& \Pi_{y z}=p_{o} \frac{\partial v_{z}}{\partial z} \approx-p_{o} \frac{v_{i}}{d}
\end{aligned}
$$

The essence of the diffusion region is that the plasma is unmagnetized, i.e., that it does not obey an $E x B$ drift. Accordingly, we may ignore the convective $(\sim v \mathrm{x} B)$ term in (12). In addition, it is reasonable to assume that no $y$ directed momentum flux enters the diffusion region from above or below.

We can now integrate (12) over one quarter of the diffusion region. Using (13) and ignoring the inflow of $y$-directed momentum, we obtain:

$$
n_{o} E^{*} d L-p_{o}\left(\frac{v_{o}}{B_{o}} \frac{d}{L}+v_{i} \frac{L}{d}\right)=-n_{o} v_{y} v_{o} d
$$

Eqn. (14) shows that the accelerative force of the reconnection electric field (first term) is balanced by quasi-viscous dissipation (second term), and by the loss of momentum through the outflow boundary (term on the RHS).

Insertion of the (2), and expressing the product of density and velocity by the current density (8) yields:

$$
n_{o}{ }^{2} d-p_{o}\left(\frac{1}{B_{o} L}+\frac{v_{i}}{v_{o}} \frac{L}{d^{2}}\right)=\frac{1}{2}\left(\frac{1}{d}-n_{o} \frac{d}{L^{2}}\right)
$$


This is formally solved for $\mathrm{d}$ :

$$
d^{2}=\frac{1}{2}\left[2 p_{o}\left(\frac{1}{n_{o}}+n_{o}\right)+1-n_{o} \frac{d^{2}}{L^{2}}\right] \frac{1}{n_{o}^{2}}
$$

Eqn. (20) shows that the diffusion region thickness is controlled by the compression ratio (lower inflow/outflow density ratio implies smaller sheet thickness) and current density (where larger current density implies a thicker current layer).

\section{ENERGY EQUATION}

The energy equation describes how energy inflow is converted to energy outflow, in form of enthalpy, Poynting-, and kinetic energy flux. Using assumptions 4) and 11), we find, for each species, an energy equation of the form (see the appendix):

$$
\stackrel{r}{v} \cdot \nabla p=-\frac{5}{3} p \nabla \cdot \stackrel{r}{v}-\frac{2}{3}(\stackrel{t}{\Pi} \cdot \nabla) \cdot \stackrel{r}{v}
$$

In order to represent some effects of anisotropy as well as some heat flux effects we generalize this equation slightly by means of a polytropic index $\gamma$ :

$$
\stackrel{r}{v} \cdot \nabla p=-\not p \nabla \cdot \stackrel{r}{v}-(\gamma-1)(\stackrel{\imath}{\Pi} \cdot \nabla) \cdot \stackrel{r}{v}
$$

Eqn. (22) shows how the magnetohydrodynamic, resistive heating term $(\gamma-1) \eta j^{2}$ is

replaced by a kinetic, quasi-viscous, dissipation term. A substitution of the form $u=p^{1 / \gamma}$ and an expansion of the last term (see appendix) provides further simplification: 


$$
\nabla \cdot(u \stackrel{r}{v})=-\frac{\gamma-1}{\gamma} u^{1-\gamma}\left(\Pi_{y z} \frac{\partial v_{y}}{\partial z}+\Pi_{y x} \frac{\partial v_{y}}{\partial x}\right)
$$

Assumption 10) lets us neglect the $x$ derivative of the current-directed velocity. Then integration over one box quarter provide:

$$
v_{o} u_{o} d=-\frac{\gamma-1}{\gamma} u_{o}^{1-\gamma} \int d x d z \Pi_{y z} \frac{\partial_{y}}{\partial z}+v_{i} u_{i} L
$$

Again, we will, without loss of generality, consider the electron species. Using (13) and (3) we find:

$$
d=-\frac{\gamma-1}{\gamma} v_{y} n_{o}+\frac{v_{i}}{v_{o}} \frac{u_{i}}{u_{o}} L
$$

where we have used the equality

$$
u_{o}^{-\gamma}=\frac{1}{p_{o}}
$$

We use (8) to relate the current density to $n_{o} v_{y}$ as well as the continuity equation (1) to obtain another equation for $d^{2}$ :

$$
d^{2}\left(1-n_{o} \frac{u_{i}}{u_{o}}\right)=\frac{\gamma-1}{2 \gamma}\left(1-n_{o} \frac{d^{2}}{L^{2}}\right)
$$

Eqn. (26) provides another estimate for the sheet thickness. In the following section, we will combine estimates (6) and (20) for this thickness from the momentum equation with (26) to derive a set of equations, which, among others, specify the reconnection rate. If the kinetic energy in the inflow is ignored, these equations can be solved analytically. 
As we will see, inclusion of the inflow kinetic energy is necessary. The equations are then solved by a simple iteration.

\section{SOLUTION}

In this section, we combine results from previous sections for estimates of reconnection rates and other significant parameters. For this purpose, we insert the RHS of (19) into (26). Sorting then yields:

$$
d^{2}\left(1-n_{o} \frac{u_{i}}{u_{o}}-n_{o}{ }^{2} \frac{\gamma-1}{\gamma}\right)=-\frac{\gamma-1}{\gamma} \frac{p_{o}}{n_{o}}\left(\frac{1}{n_{o}}+n_{o}\right)
$$

The unknowns in this equation are the diffusion region parameters thickness $d$, density $n_{o}$ and pressure $p_{o}=u_{o}^{\gamma}$. We can use assumption 6) to remove the thickness. Using (6) yields thus:

$$
1-n_{o} \frac{u_{i}}{u_{o}}-n_{o}{ }^{2} \frac{\gamma-1}{\gamma}=-\frac{\gamma-1}{2 \gamma}\left(\frac{1}{n_{o}}+n_{o}\right)
$$

We define for convenience:

$$
\varepsilon=\frac{u_{i}}{u_{o}}=\left(\frac{p_{i}}{p_{o}}\right)^{1 / \gamma}
$$

Then (28) can be rewritten as an equation for $n_{o}$ : 


$$
n_{o}^{3}+\left(\frac{\gamma}{\gamma-1} \varepsilon-\frac{1}{\kappa}\right) n_{o}{ }^{2}-\frac{\gamma}{\gamma-1} n_{o}-\frac{1}{2}=0
$$

This equation can be solved by standard techniques. Furthermore, it is evident that (30) has at least one solution $n_{o}>0$. However, the solution still parametrically depends on the inflow total pressure, which depends on the inflow ram pressure. An analytical solution is possible only if the ram pressure is negligible; in the more general case, a solution is found by simple iteration.

The next step is to determine the aspect ratio of the diffusion region. A suitable expression can be found by combining the energy equation (26) with the bounce width (6):

$$
\frac{4 \gamma}{\gamma-1} \frac{p_{o}}{n_{o}}\left(1-n_{o} \varepsilon\right)=\left(1-n_{o} \frac{d^{2}}{L^{2}}\right)
$$

This is readily solved for $d / L$ :

$$
\frac{d}{L}=\left[\frac{1}{n_{o}}-\frac{4 p_{o} \gamma}{\gamma-1} \frac{1-n_{o} \varepsilon}{n_{o}{ }^{2}}\right]^{1 / 2}
$$

It is noteworthy that this equation does not always have a real, i.e., physical, solution. This is the case if the required change of specific entropy $\left[\sim(u / n)^{\gamma}\right]$ from inflow to outflow is too large to be provided by the available quasi-viscous heating.

Using the outflow velocity (10) combined with (3), the reconnection electric field (2) is obtained from the above results: 


$$
E^{*}=\frac{1}{2}\left(1-n_{o} \frac{d^{2}}{L^{2}}\right)^{1 / 2} n_{o} \frac{d}{L}
$$

As noted above, solving these equations requires iterations, since $\varepsilon$ depends on the inflow velocity and the diffusion region aspect ratio. The solution process is thus as follows: As an initial assumption, we ignore the inflow velocity and the aspect ratio in (4). We solve eqns. (30)-(33), and then recalculate $p_{o}$ and $\varepsilon$. We then restart the cycle. The process is repeated until convergence has been achieved, which typically takes only about 20 iterations.

\section{RESULTS}

Results for the reconnection electric field are shown in Figure 1, as a function of polytropic index. The different graphs represent different values of the plasma pressure parameter $\beta=4 \mu_{o} p_{i} / B_{i}^{2}$ in the inflow region.

Except for the case of vanishing pressure in the inflow region, all graphs predict a maximum reconnection rate of

$$
E_{\max }^{*} \approx 0.4
$$

irrespective of the values of $\beta$. For smaller values of $\beta$, solutions exist only for polytropic indices somewhat larger than adiabatic values. The lack of solutions for smaller values of the polytropic index is caused the required amount of heating, which is larger for smaller inflow pressure. The heating term in (22) is proportional to $\gamma-1$, 
Therefore, larger values of the polytropic index are required for lower inflow pressure to assure the existence of solutions.

Conversely, large values of the polytropic index represent lower compressibility. It is likely that the dynamics of an inflowing cold plasma is more complex than described by a polytropic pressure law, and likely involves the interaction and partial thermalization of counterstreaming beams. Such anisotropies are not included in this model, but the associated, quasi-one dimensional compression would be qualitatively represented by a larger polytropic index.

The maximum reconnection rate is approximately twice that reported, for example in the GEM challenge results. An obvious contender for explaining the differences is the fact that the kinetic GEM challenge calculations were performed for mass ratios other than unity. Another possible reason for this discrepancy lies in the adopted scaling. Whereas the GEM challenge results ${ }^{15}$, as well as most studies in the literature, are scaled by the asymptotic Alfven speed and magnetic field, the scaling adopted here is by the values of Alfven speed and magnetic field at the edge of the diffusion region. These values are typically reduced from their asymptotic values. In fact, a recent study of the electron diffusion region in a simulations with mass ratio 25 found similar values of the reconnection electric field if the scaling parameters were taken from the diffusion region edge $^{16}$. Future studies will determine how results obtained for mass ratios other than unity relate to the ones in this study.

Furthermore, it should be noted that the maximum rate shown here may not be assumed in reality or in simulations. In order for the system to exhibit a rate of $E^{*}=0.4$, it 
has to exhibit a very specific level of compressibility. In reality, the plasma may be better described by a different value of the polytropic index, e.g., by its adiabatic value. Since the theory treats the polytropic index as a free parameter it cannot predict which value would actually be obtained. In this broad sense our results should be seen as consistent with typical values of the reconnection electric field in the literature.

The ratio of outflow to inflow density $n_{o}$ is shown in Figure 2 . The density increase exhibits the expected behavior: We find larger increases for smaller pressure in the inflow region, as well as larger increases for smaller values of the polytropic index. The physical reason behind these tendencies is that less compression is necessary for higher inflow pressure or larger polytropic index to provide current sheet pressure in addition to that obtained from nonideal heating.

The last term of the RHS of (22) constitutes nonadiabatic heating, i.e., a deviation from entropy conservation. This deviation can be measured by the ratio

$$
S=p_{i} n_{o}^{\gamma} / p_{o} n_{i}^{\gamma}
$$

which compares the specific entropies in inflow and outflow regions. This parameter is shown in Figure 3. It is evident from the figure that the specific entropy in the outflow is considerably larger than in the inflow. Specifically, we find larger entropy increases (and, accordingly, more nonadiabatic heating), for smaller values of the pressure in the inflow region. Increasing the polytropic index produces larger values of S, consistent with the notion that adiabatic compression will become more important for larger values of $\gamma$. 
The dimensions of the diffusion region vary with inflow and compressibility parameters. For example, Figure 4 displays the diffusion region thickness, determined by the bounce width (6). Here we find single maxima of $d$, and, as expected, generally larger values for higher inflow pressure.

The diffusion region aspect ratio is shown in Figure 5. All curves start near zero for lower polytropic indices, and terminate at rather large aspect ratios. For fixed $\gamma$ we find more oblong shape of the diffusion region for smaller inflow pressure. In a compressible plasma as discussed here the aspect ratio is not directly related to the reconnection rate. Furthermore, a large aspect ratio involves larger outflow magnetic fields, and consequently a lower current density and energy conversion rate. The combination of these factors leads to the single peak of the reconnection rate displayed in Fig. 1 rather than a rate peak for the largest aspect ratio.

The outflow velocity is shown in Figure 6. Here we find a trend of larger outflow velocities for lower inflow pressure, and for lower values of the polytropic index. A comparison with Fig. 5 demonstrates that larger outflow speeds are also related to more oblong shapes of the diffusion region, reflecting the impact of the continuity equation.

For comparison with an alternative way of scaling used by many MHD modelers we express the outflow velocity in units of the (single-species) outflow Alfven speed, defined by

$$
v_{A o}=\frac{B_{i}}{\sqrt{\mu_{o} m n_{o}}}=v_{A i} \sqrt{\frac{n_{i}}{n_{o}}}
$$


Figure 7 displays the result of this scaling. It is noteworthy that all graphs provide values of order unity - a qualitative match with the simple Sweet-Parker scaling results.

The total, i.e., combined, energy flux densities in the inflow region are in dimensionless form:

Poynting flux

$$
S_{i}=v_{i}
$$

enthalpy flux

$$
\mathrm{E}_{i}=\frac{2 \gamma p_{i}}{\gamma-1} v_{i}
$$

kinetic energy flux

$$
K_{i}=v_{i}^{3}
$$

and they are dominated by Poynting flux. A typical example is shown in Figure 8, which depicts their variation with adiabatic index for inflow $\beta=0.3$. Enthalpy and kinetic energy fluxes are of considerably smaller magnitude, with the Poynting flux exceeding the others by factors between two and five. The dominance of the Poynting flux is a manifestation of the predominant energy conversion: from magnetic energy to plasma internal and kinetic energies.

This conversion is further illustrated in Figure 9, which depicts the outflow energy flux densities, again for inflow $\beta=0.3$. These energy flux densities are given by:

Poynting flux 


$$
S_{o}=E^{*} B_{o}
$$

enthalpy flux

$$
\mathrm{E}_{o}=\frac{2 \gamma p_{o}}{\gamma-1} v_{o}
$$

kinetic energy flux

$$
K_{o}=n_{o} v_{o}{ }^{3}
$$

We see a dominance of outflow enthalpy flux over kinetic energy flux and Poynting fluxes, a fact not represented in traditional Sweet-Parker models ${ }^{17}$. The increase of Poynting flux for more incompressible conditions is a consequence of the larger aspect ratio of the diffusion region, which is associated with a larger value of the outgoing magnetic field. The lower outflow velocity leads to an overall reduced kinetic energy flux for larger values of $\gamma$.

The dominance in the outflow of the enthalpy flux over the other two energy fluxes is typical, as is seen in Figures 10 and 11. Here we see that for all parameters of compressibility and inflow pressure the enthalpy flux dominates over kinetic energy flux by factors between two and four, and over Poynting flux by factors of two to five.

An exception is the zero inflow pressure case, where enthalpy and kinetic energy flux are comparable. This is clearly a consequence of the singular, zero inflow pressure, condition, where no pressure can be obtained by compressing the inflowing plasma. Accordingly, the outflow enthalpy flux is somewhat smaller than those found for finite inflow pressure, even if the latter is quite small. The relatively large kinetic energy flux in 
this case results from a combination of relatively fast outflow velocity (Fig. 6) combined with a larger current sheet density (Fig. 2). Because of the very special conditions inherent in the zero-pressure model, we feel that this model represents an exceptional case, which is likely not typical of reconnection processes.

\section{SUMMARY}

In this paper, we developed an analytical theory of collisionless magnetic reconnection in a symmetrical pair-plasma system. We started by identifying the basic functions of the reconnection electric field: maintaining both the current density and the pressure in the reconnection diffusion region. Combining these basic concepts with a set of simplifying assumptions, we could use the moment equations to derive balance equations for mass, momentum or current density, and internal energy. In both energy and momentum equations, we implemented a dissipation model, which is derived from the evolution of the full particle pressure tensor, where only heat flux is neglected. This model appears to be consistent with a number of recent numerical simulations ${ }^{9-12}$. This dissipation model introduces irreversibility into the physical process. In order to mimic some effects of heat flux, we replaced the adiabatic index by a more general polytropic index, the variation of which also describes different compressibilities.

Assuming a current sheet width determined by the bounce excursions of particles residing within the current layer, we succeeded in combining the set of equations into an expressions for the reconnection electric field and other, related, parameters. A solution of this system was obtained from a simple iterative procedure. 
The solutions featured a number of expected properties: Density increases from inflow to outflow, pressure increases through a combination of adiabatic compression and nonadiabatic, quasi-viscous, heating, dominance of enthalpy flux in the outflow, and an outflow speed comparable to the Alfven speed based on outflow density and inflow magnetic field.

The surprising result, however, is that we found a peak reconnection rate of approximately $E^{*} \approx 0.4$, which is quite independent on the inflow parameters. This number is larger than the rates of 0.1-0.2 typically found in kinetic or Hall-MHD models - even though Hall-MHD approaches are not kinetic in nature. However, the rate obtained here is based on a normalization to magnetic field and density at the inflow edge of the diffusion region, the value of which was recognized earlier ${ }^{18}$. Here magnetic fields and Alfven speeds are usually reduced from the asymptotic values used to normalize kinetic and fluid numerical models. A recent investigation, which normalized the reconnection electric field to parameters as the edge of the electron diffusion region, found values similar to the ones here of the scaled electric field ${ }^{16}$. Accounting for the expected reductions of the product of Alfven speed and magnetic field, the rate found here is therefore qualitatively consistent with kinetic, numerical modeling.

Within the scope of our theory, we have therefore shown that there is a limit to the value of the scaled reconnection electric field. It should be noted that the actual value may be different from the one derived here - the present theory had to employ simplifying assumptions and quantitative predictions should be seen in this light. Future investigations will extend this theory to different particle masses, to configurations with guide magnetic field, and perhaps employ more complex descriptions of particle 
pressure. It may also be possible to connect this solution to a suitable outer solution. These will be challenges to be addressed as the next steps. For now, we hope that the present investigation provides some hope that the longstanding issue of the magnitude of the reconnection rate may be amenable to a solution after all.

\section{ACKNOWLEDGEMENTS}

This research was supported by NASA's MMS mission. One of us (SZ) gratefully acknowledges support from NASA's postdoctoral program. The authors acknowledge helpful comments from Karl Schindler. 


\section{APPENDIX: DERIVATION OF THE PRESSURE EQUATION}

The pressure tensor evolution equation is:

$$
\frac{\partial \dot{P}}{\partial t}=-\nabla \cdot(\stackrel{\mathrm{t}}{\mathrm{P}})-\stackrel{\mathrm{t}}{P} \cdot \nabla \stackrel{\mathrm{r}}{v}-[\stackrel{\mathrm{t}}{P} \cdot \nabla \stackrel{\mathrm{r}}{v}]+\frac{q}{m}(\stackrel{\mathrm{t}}{P} \times \stackrel{\mathrm{r}}{B}+[\stackrel{\mathrm{t}}{P} \times \stackrel{\mathrm{r}}{B}])-\nabla \cdot \stackrel{\mathrm{t}}{Q}
$$

The total internal energy of the plasma species under consideration is:

$$
K:=\frac{1}{2} \operatorname{Trace}(\stackrel{\mathrm{t}}{P})
$$

From this we obtain:

$$
\frac{\partial K}{\partial t}=-\nabla \cdot(\stackrel{r}{v} K)-\frac{1}{2} \operatorname{trace}(\stackrel{\mathrm{t}}{P} \cdot \nabla \stackrel{\mathrm{r}}{v})-\frac{1}{2} \operatorname{trace}\left[\stackrel{\mathrm{t}}{P} \cdot \nabla v^{\mathrm{r}}\right]-\frac{1}{2} \operatorname{trace}(\nabla \cdot \stackrel{\mathrm{t}}{Q})
$$

We define a heat flux vector by:

$$
q_{i}=\frac{1}{2} \sum_{k} Q_{i k k}
$$

Insertion and evaluation of the traces in (A3) yields:

$$
\frac{\partial K}{\partial t}==-\sum_{i, k} P_{i k} \frac{\partial}{\partial x_{k}} v_{i}-\nabla \cdot(\stackrel{r}{v} K)-\nabla \cdot \stackrel{r}{q}
$$


The pressure is now defined as $p=2 K / 3$. If we ignore the divergence of the heat flux, we obtain:

$$
\frac{\partial p}{\partial t}=-\frac{2}{3} \sum_{i, k} P_{i k} \frac{\partial}{\partial x_{k}} v_{i}-\nabla \cdot\left({ }_{v p}^{r}\right)
$$

Assumption 5 suggests to write:

$$
\grave{P}=p \grave{1}+\grave{\Pi}
$$

Insertion into (A6) yields:

$$
\frac{\partial p}{\partial t}=-\stackrel{r}{v} \cdot \nabla p-\frac{5}{3} p \nabla \cdot \stackrel{r}{v}-\frac{2}{3}(\stackrel{t}{\Pi} \cdot \nabla) \cdot \stackrel{r}{v}
$$

Finally, the pressure equation results from expanding the last term in (A8) and using the symmetry of the pressure tensor and the assumed translational invariance:

$$
\frac{\partial p}{\partial t}=-\stackrel{r}{v} \cdot \nabla p-\frac{5}{3} p \nabla \cdot \stackrel{r}{v}-\frac{2}{3}\left(\Pi_{y z} \partial_{z} v_{y}+\Pi_{x y} \partial_{x} v_{y}\right)
$$




\section{REFERENCES}

${ }^{1}$ H. E. Petschek, in AAS/NASA Symposium on the Physics of Solar Flares,

W. N. Ness, Ed. (NASA, Washington, DC, 1964), p. 425.

${ }^{2}$ V. M. Vasyliunas, Rev. Geophys. Spac. Phys. 13, 303 (1975).

${ }^{3}$ P. A. Cassak and M. A. Shay, Phys. Plasmas 14, 102114 (2007)

${ }^{4}$ P. A. Cassak and M. A. Shay, Phys. Plasmas 16055704 (2009)

${ }^{5}$ J. Borovsky and M. Hesse, Phys. Plasmas 14, 102309 (2007).

${ }^{6}$ J. Birn, J. E. Borovsky, and M. Hesse, Phys. Plasmas 15, 032101 (2008).

${ }^{7}$ L. Chacón, A. N. Simakov, V. S. Lukin, and A. Zocco, Phys. Rev. Lett., 101, 025003 (2008).

${ }^{8}$ A. N. Simakov, and L. Chacón, Phys. Plasmas 16, 055701 (2009).

${ }^{9}$ N. Bessho and A. Bhattacharjee, Phys. Rev. Lett. 95, 245001 (2005).

${ }^{10}$ N. Bessho and A. Bhattacharjee, Phys. Plasmas 14, 056503 (2007)

${ }^{11}$ W. Daughton and H. Karimabadi, Phys. Plasmas 14, 072303 (2007)

${ }^{12}$ M. Swisdak, Y-H. Liu, and J. F. Drake, Astrophys. J. 680, 999 (2008).

${ }^{13}$ D. Tsiklauri, Phys. Plasmas 15, 112903 (2008).

${ }^{14}$ M. Kuznetsova, M. Hesse, and D. Winske, J. Geophys. Res. 103, 199 (1998).

${ }^{15}$ J. Birn, J. F. Drake, M. A. Shay et al., J. Geophys. Res. 106, 3715 2001. 
${ }^{16}$ A. Klimas, M. Hesse, and S. Zenitani, Phys. Plasmas 15, 082102 (2008).

${ }^{17}$ E. N. Parker, J. Geophys. Res., 62, 509 (1957).

${ }^{18}$ M. A. Shay et al., Phys. Plasmas 11, 2199 (2004). 


\section{Figure captions}

Figure 1 (color online). Reconnection electric field depending on inflow plasma $\beta$ and polytropic index $\gamma$.

Figure 2 (color online). Ratio of outflow and inflow density, depending on inflow plasma $\beta$ and polytropic index $\gamma$.

Figure 3 (color online). Entropy ratio $p_{i} n_{o}^{\gamma} / p_{o} n_{i}^{\gamma}$ depending on inflow plasma $\beta$ and polytropic index $\gamma$.

Figure 4 (color online). Diffusion region thickness $d$ depending on inflow plasma $\beta$ and polytropic index $\gamma$.

Figure 5 (color online). Diffusion region aspect ratio $d / L$ depending on inflow plasma $\beta$ and polytropic index $\gamma$. 
Figure 6 (color online). Outflow velocity depending on inflow plasma $\beta$ and polytropic index $\gamma$.

Figure 7 (color online). Outflow velocity based on outflow density depending on inflow plasma $\beta$ and polytropic index $\gamma$.

Figure 8 (color online). Inflow energy flux densities for upstream $\beta=0.3$.

Figure 9 (color online). Outflow energy flux densities for upstream $\beta=0.3$.

Figure 10 (color online). Outflow kinetic energy flux density plotted versus enthalpy flux density for all parameters. The lower fluxes are obtained for larger values of the polytropic index $\gamma$, with the exception of the $\beta=1$ calculation. Here, lower values of $\gamma$ yield larger kinetic energy but lower enthalpy flux densities.

Figure 11 (color online). Outflow Poynting flux density plotted versus enthalpy flux density for all parameters. Here higher Poynting fluxes are obtained for smaller values of 
the polytropic index $\gamma$, again with the exception of the $\beta=1$ calculation. Here, lower values of $\gamma$ yield larger kinetic energy but lower enthalpy flux densities. 


\section{outflow/inflow density ratio}

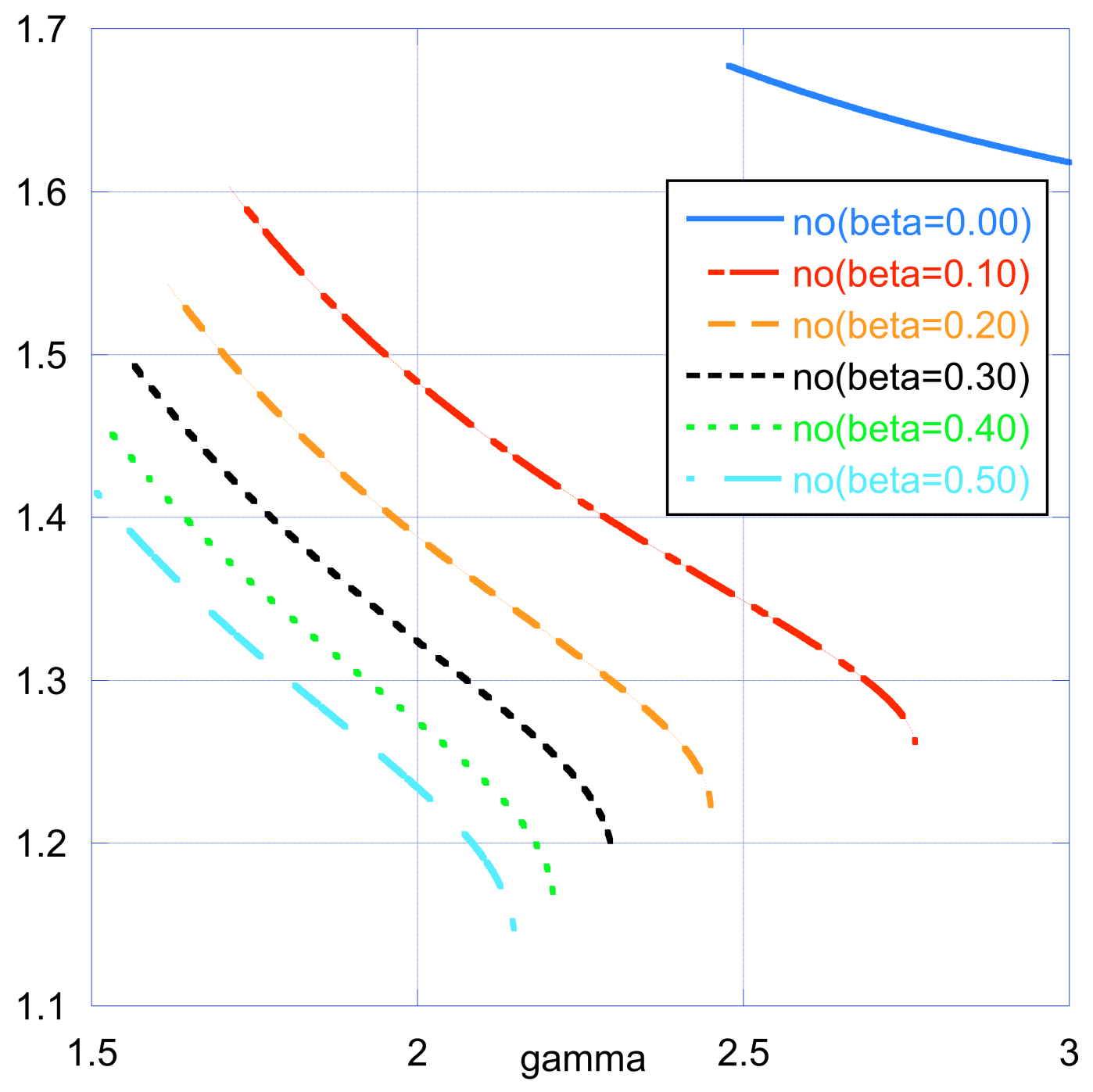




\section{inflow/outflow entropy ratio}

0.7

0.6

0.4

0.3

0.1

0 


\section{diffusion region aspect ratio}

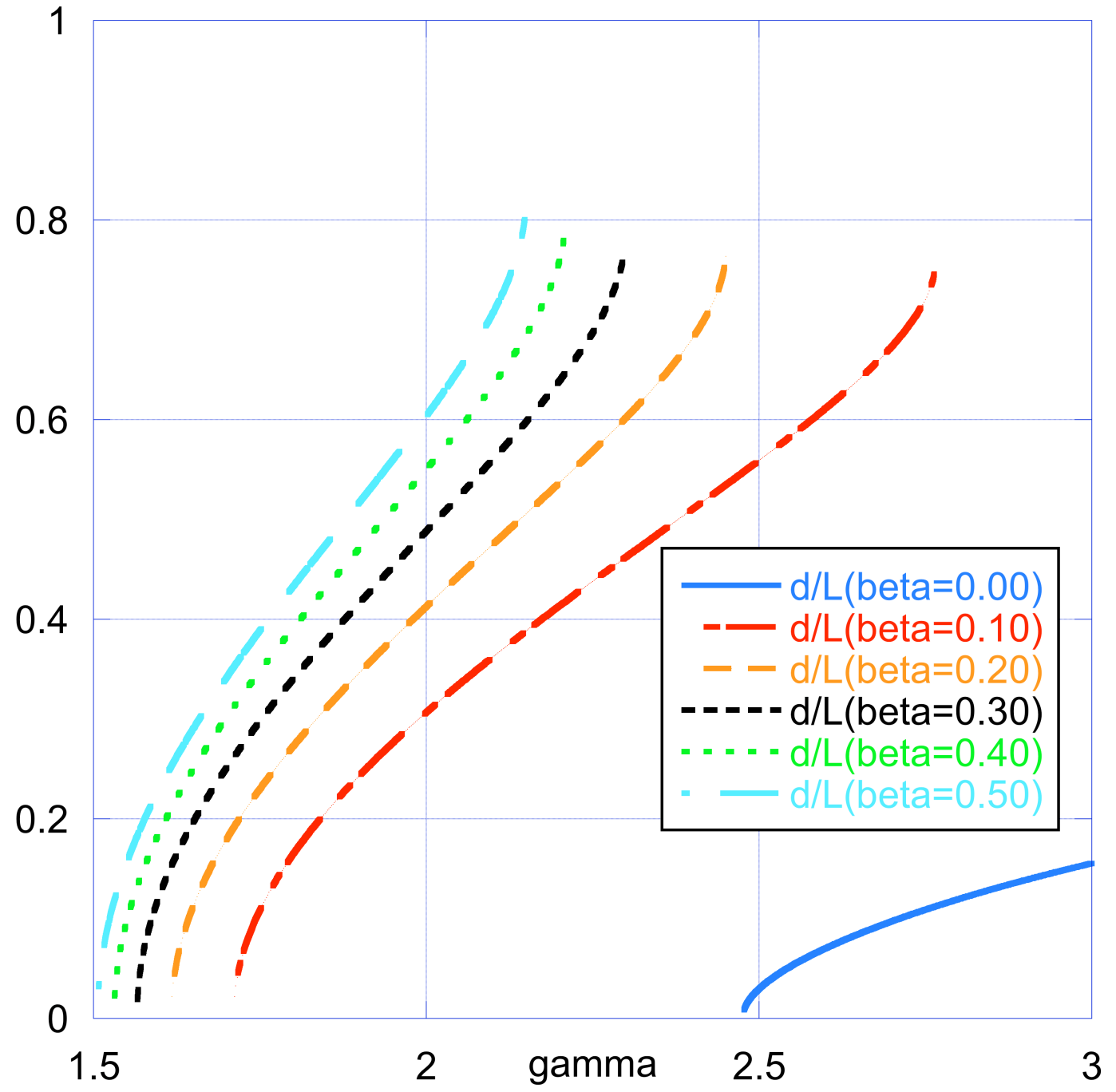




\section{outflow velocity}

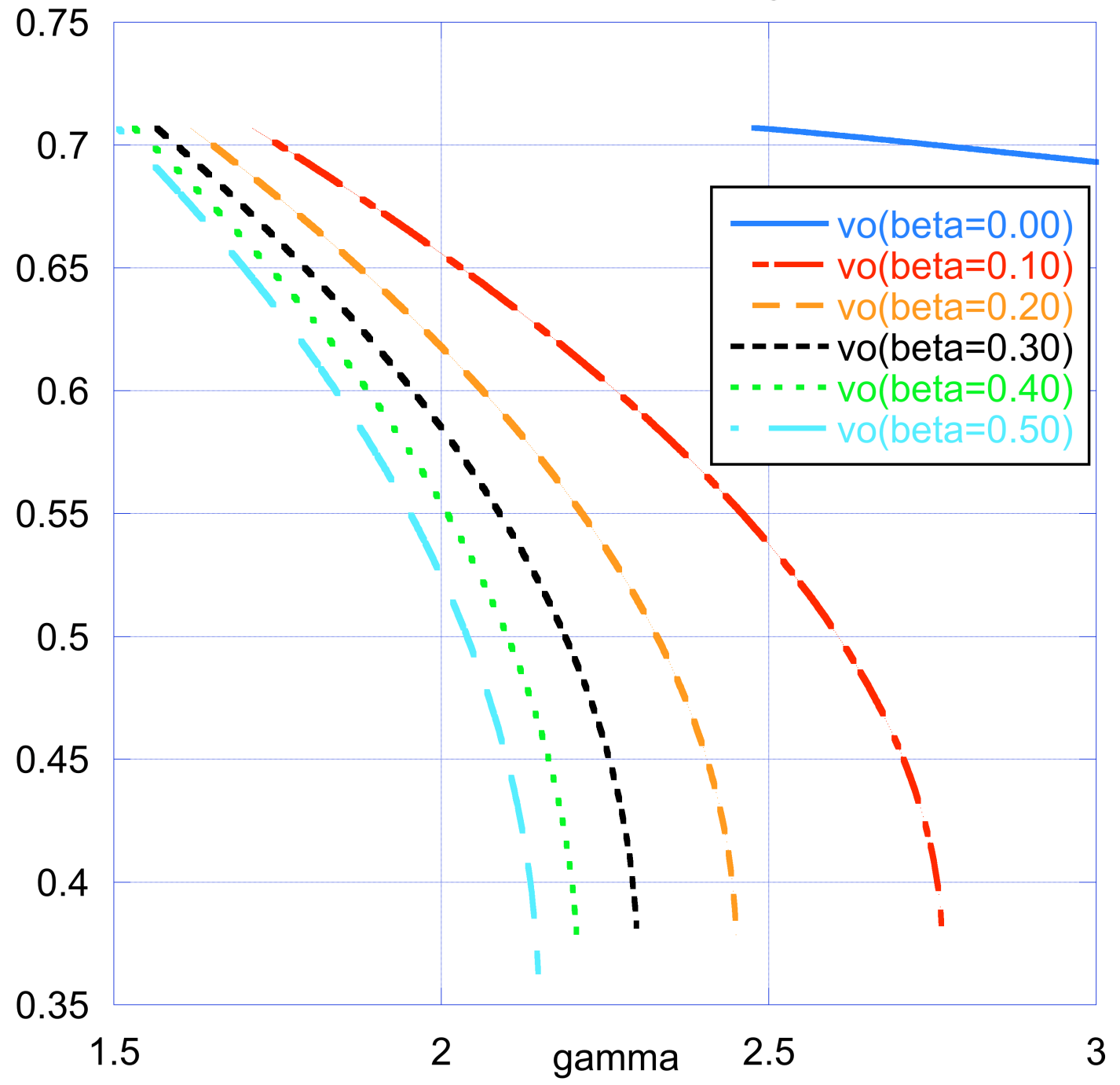




\section{outflow velocity in units of outflow Alfven speed}

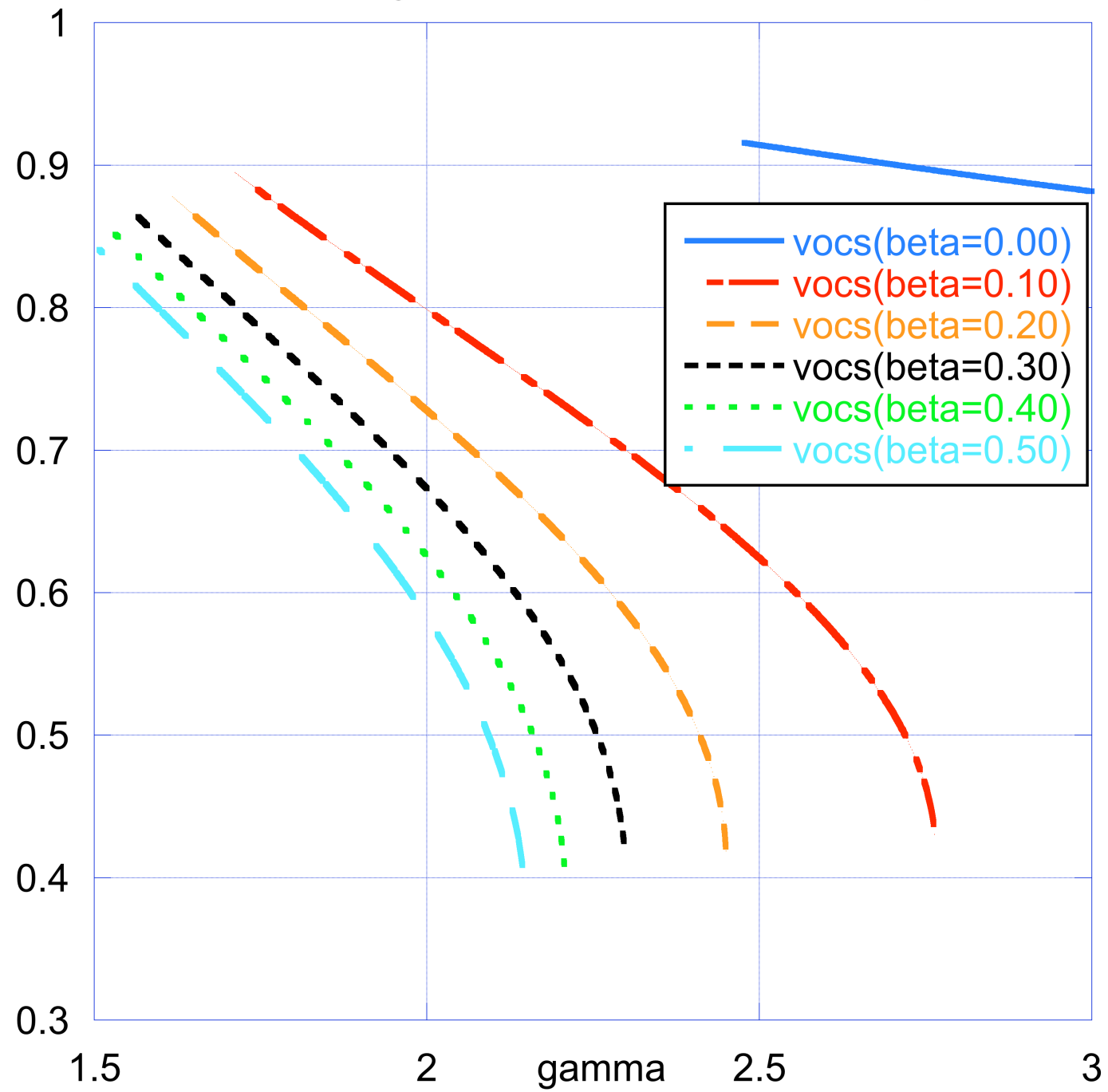




\section{outflow kinetic energy vs. outflow enthalpy flux}

0.6

0.5

0.4

0.3

0.2

0.1
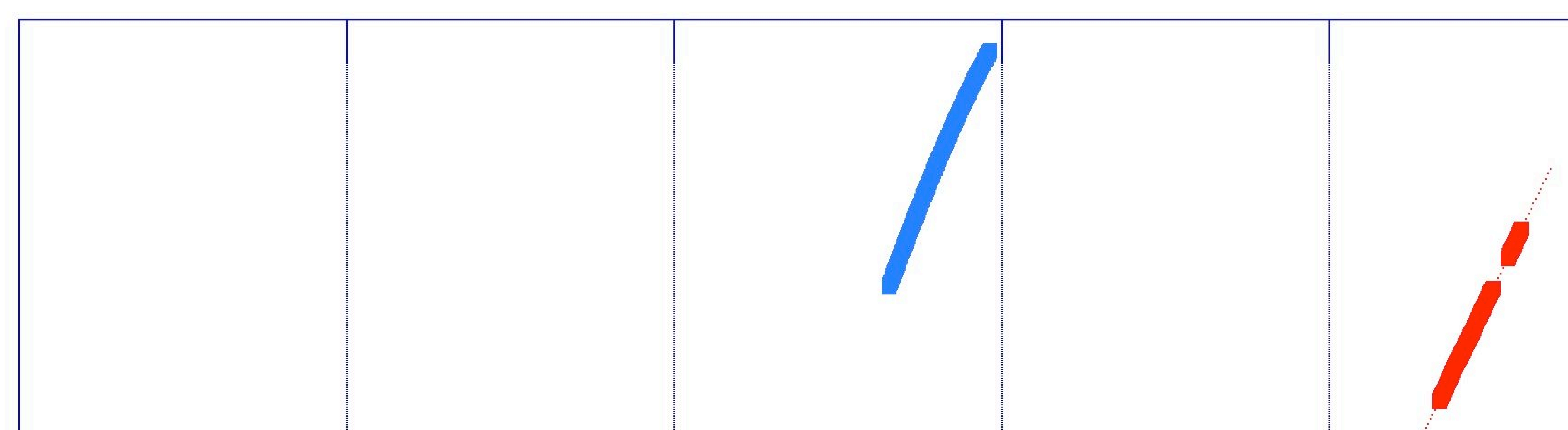


\section{outflow Poynting flux vs outflow enthalpy flux}

0.35

0.25

0.15

0.2

0.05

0.1

0
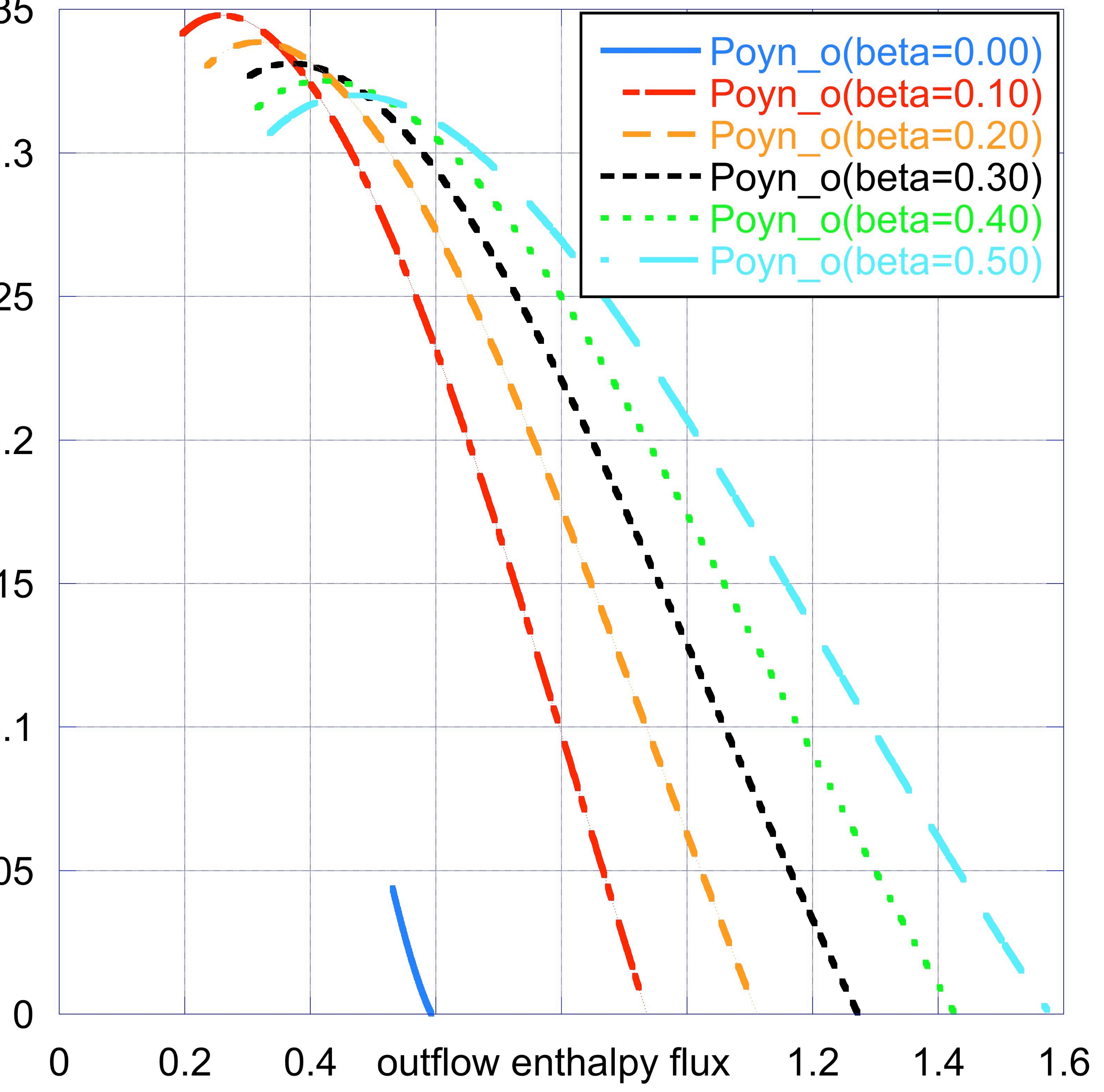